\title{
Determination of Soft X-ray Emission Spectroscopy Parameters using Experimental Data for Quantitative Microanalysis
}

\author{
Hendrix Demers ${ }^{1}$, Colin M. MacRae ${ }^{2}$, Nicholas C. Wilson ${ }^{2}$, Pierre Hovington ${ }^{3}$, Vladimir Timoshevskii ${ }^{3}$, \\ Raynald Gauvin ${ }^{1}$, and Karim Zaghib ${ }^{3}$ \\ 1. Department of Mining and Materials Engineering, McGill University, Montreal, Quebec, Canada. \\ 2. CSIRO Mineral Resources, Clayton, VIC, Australia. \\ 3. Hydro-Quebec Research Institute, Varennes, Quebec, Canada.
}

Recent development in X-ray detection technology like the windowless energy dispersive spectrometer silicon drift detector (EDS SDD) [1] and the soft x-ray emission spectrometer (SXES) [2] allow the measurement of soft x-ray lines $(>50 \mathrm{eV})$. However, the conventional microanalysis models are not well tested for these soft x-ray lines. Furthermore, chemical bounding can affect the x-ray emission [3], which are not currently taken into account with the conventional models. The relationship between emitted xray intensity and accelerating voltage was noted by Andersen (1967) and Kyser (1971) use it to experimentally measured mass absorption coefficients (MACs). In this work, soft x-ray emission parameters like the MAC are determined by comparing experimental measurements and Monte Carlo simulations [4] using the emitted x-ray intensity and accelerating voltage relationship.

The Monte Carlo simulations allow to understand the effect of one parameter on the soft x-ray emission. For example, Figure 1 show the variation of emitted intensity for $\mathrm{Li} \mathrm{K}_{\alpha}$ in metallic lithium sample with accelerating voltage for different mass absorption values. The typical variation of emitted x-ray intensity with accelerating voltage is a sharp increase at low accelerating voltage, a maximum emitted intensity for a specific voltage and slow intensity decrease at higher voltage. The specific accelerating voltage of the maximum emitted intensity is strongly dependant on the MAC value in the sample. Larger MAC reduces both the specific voltage and the maximum intensity. Furthermore, the curvature of the high voltage decrease is increased by larger MAC. However, the sharp increase at low accelerating voltage is not affected by the MAC value. Figure 2 shows experimental emitted $\mathrm{x}$-ray intensities for $\mathrm{Li} \mathrm{K}_{\alpha}$ in metallic lithium sample measured with a SXES on a JEOL 8530 field emission microprobe and their comparison with Monte Carlo simulations. The agreement between the experiment and the simulation is close but not perfect when using the nominal value of MAC $\left(6060 \mathrm{~cm}^{2} / \mathrm{g}\right)$ taken from Chantler (2005) tabulation [5]: the specific voltage is different. Using the generated $\varphi(\rho z)$ distribution obtained by Monte Carlo and the experimental data, a better MAC value of $8000 \mathrm{~cm}^{2} / \mathrm{g}$ was calculated. The same specific voltage was obtained, but the maximum emitted intensity still differ. To understand why, the effect of each soft x-ray emission parameter was studied by Monte Carlo simulation and compared to experimental data from pure element and compound samples using SXES and windowless EDS SDD. These results should allow the determination of the parameter values needed for soft x-ray quantitative microanalysis.

References:

[1] S. Burgess, X. Li, and J. Holland, Microscopy and Analysis 27 (2013), p. S8-S13.

[2] M. Terauchi et al, Journal of Electron Microscopy 61 (2012), p. 1-8.

[3] P. Hovington et al, Scanning (2016), doi: 10.1002/sca.21302.

[4] R. Gauvin and P. Michaud, Microscopy and Microanalysis 15 (2009), p. 488.

[5] C. Chantler et al, (2005), [Online] Available: http://physics.nist.gov/ffast. 


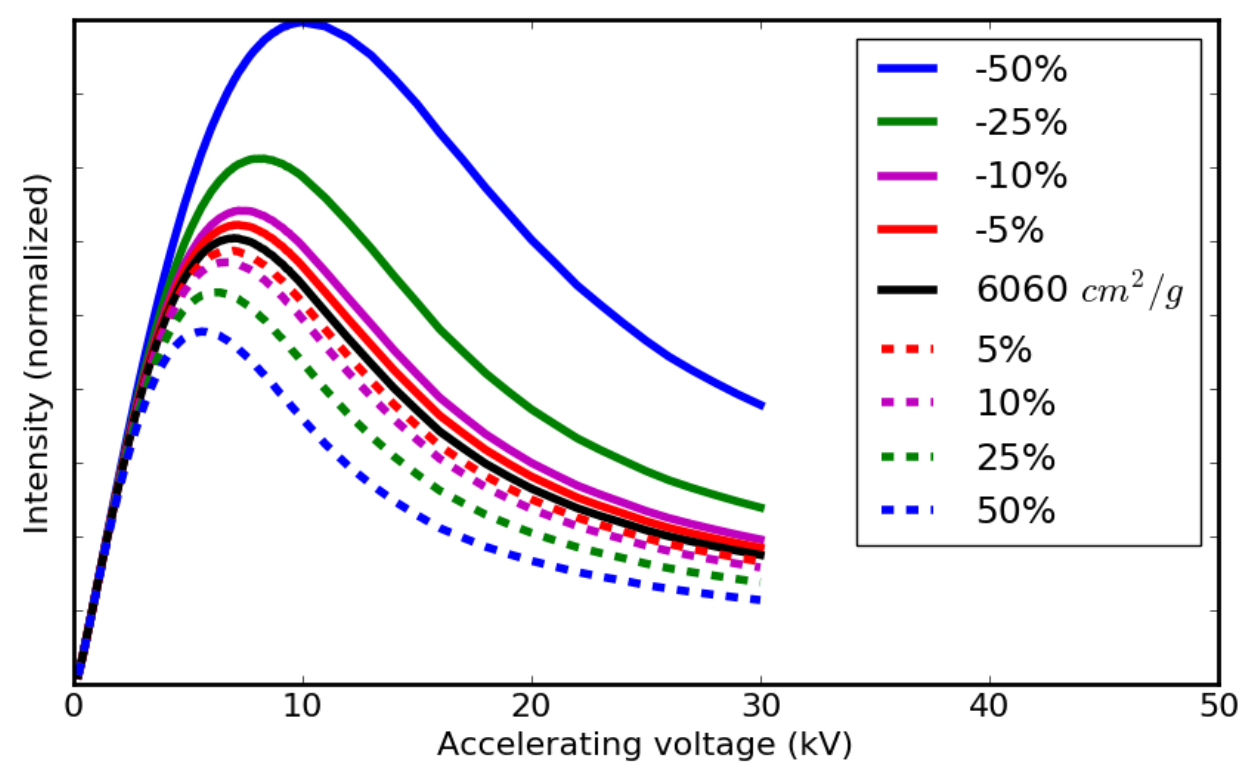

Figure 1. Variation of emitted intensity for $\mathrm{Li} \mathrm{K}_{\alpha}$ in metallic lithium sample with accelerating voltage for different mass absorption values. The intensity was simulated with MC X-ray [4] and the nominal value of MAC $\left(6060 \mathrm{~cm}^{2} / \mathrm{g}\right)$ was taken from Chantler (2005) tabulation [5].

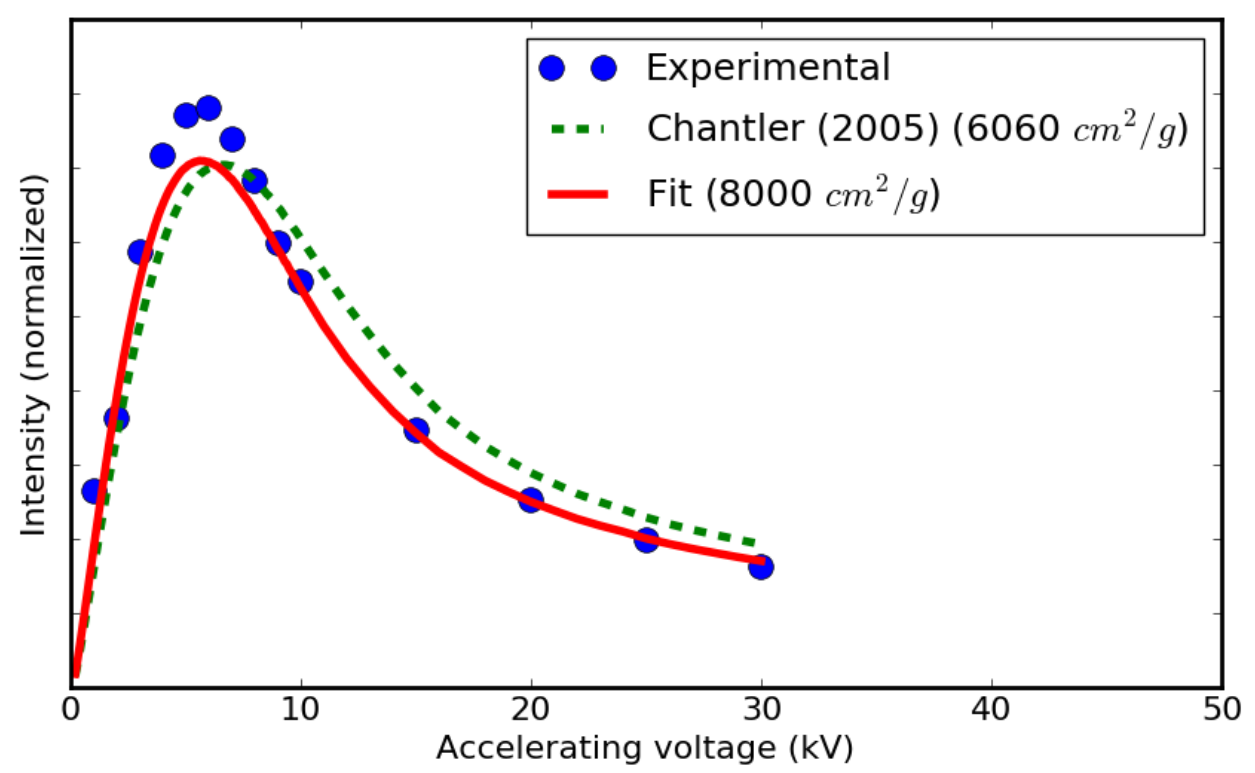

Figure 2. Variation of emitted intensity for $\mathrm{Li} \mathrm{K}_{\alpha}$ in metallic lithium sample with accelerating voltage obtained experimentally (blue circle) using the soft x-ray emission spectrometer (SXES) [2]; from Monte Carlo simulation (green dashed-line) using MAC from Chantler (2005) [5] tabulation; from Monte Carlo simulation (green dashed-line) using MAC value of $8000 \mathrm{~cm}^{2} / \mathrm{g}$ obtained by fitting the experimental data (red line). 